\title{
Asset pricing under information with stochastic volatility
}

\author{
Bertram Düring
}

\begin{abstract}
Based on a general specification of the asset specific pricing kernel, we develop a pricing model using an information process with stochastic volatility. We derive analytical asset and option pricing formulas. The asset prices in this rational expectations model exhibit crash-like, strong downward movements. The resulting option pricing formula is consistent with the strong negative skewness and high levels of kurtosis observed in empirical studies. Furthermore, we determine credit spreads in a simple structural model.
\end{abstract}

Keywords Pricing kernel · stochastic volatility · asset pricing · option pricing · credit spreads

\section{Introduction}

Asset pricing for stocks, bonds, or derivatives is based on expectations about future cash flows and risk preferences. This view is emphasized by an equilibrium approach, the stochastic discount factor or pricing kernel approach, which goes back to Rubinstein (1976) and Brennan (1979). Another, traditional approach is to specify an asset price process exogenously and price by no-arbitrage arguments. The seminal example of this approach is Black and Scholes (1973) paper on pricing of Europeanstyle options. Bick (1987) reconciles both approaches by showing that the geometric Brownian motion is consistent with a representative investor economy and that given the representative investor maximizes a von Neumann-Morgenstern utility function over terminal wealth and the asset represents total wealth, such a stochastic process implies constant relative risk aversion.

In this paper we follow the pricing kernel approach. The pricing kernel has the important property that the forward asset price equals the expected value of the product of the terminal asset value and the pricing kernel. Mathematically, the pricing

B. Düring

Institut für Analysis und Scientific Computing, Technische Universität Wien, 1040 Wien, Austria

Tel.: +4315880110128

Fax: +43158801 10199

E-mail: bduering@anum.tuwien.ac.at 
kernel characterizes the change from the subjective probability measure $P$ to the riskneutral (or equivalent martingale) measure $Q$. It is also known as the Radon-Nikodym derivative of $Q$ with respect to $P$. In this view, any pricing problem is completely determined by the distribution of terminal asset value and the shape of the asset specific pricing kernel (ASPK). The asset specific pricing kernel is the pricing kernel conditioned on the payoffs of an asset. For a detailed discussion of the pricing kernel and the asset specific pricing kernel we refer the reader to the textbook of Cochrane (2001) as well as to the articles of Câmara (2003, 2005).

Rubinstein (1976) and Brennan (1979) make explicit assumptions on the distribution and the ASPK. More precisely, they assume a representative investor and thus the representative investor's utility function characterizes the ASPK. Similar, Black and Scholes (1973) assume that the underlying is lognormally distributed, i.e., the information process follows a geometric Brownian motion, and investors are constant relative risk averse, or the asset specific pricing kernel has constant elasticity with respect to the underlying asset (see Bick, 1987; Franke et al., 1999). These assumptions lead to simple, explicit pricing formulas. However, empirical research revealed that they are not able to explain important effects in real financial markets, e.g. the volatility smile (or skew) in option prices, serial correlation in asset returns and excess and random volatility. One approach to explain such effects was to incorporate learning effects (e.g. Campbell and Cochrane, 1999) and irrational behavior as overconfidence (e.g. Daniel et. al., 2001). Another venue of literature analyzes the effect of risk preferences. Empirical studies by Ait-Sahalia and Lo (2000), Jackwerth (2000) and Rosenberg and Engle (2002) suggest that the pricing kernel is not of the constant elasticity type.

Several papers have analyzed the impact of non-constant elasticity on option prices (see e.g. Benninga and Mayshar, 2000; Franke et al., 1999). Among others, Franke et al. (1999), Lüders and Franke (2004) and Düring and Lüders (2005) showed that declining relative risk aversion leads to serial correlation in asset returns and smile effects in option implied volatility. Düring and Lüders (2005) provided explicit, analytical option pricing formulas for ASPKs with declining elasticity, when the underlying information process follows a lognormal, log-gamma, normal or uniform, with a single risk factor. Other authors (e.g. Câmara, 2003; Schroder, 2004; Vitiello and Poon, 2006) recently also extended their approaches to alternative distributions and utility functions, where they focus on preferences and distributions which yield risk neutral valuation relationships, i.e. pricing formulas without any preference parameter.

However, all these models only include a single risk factor. In real financial markets, not only asset returns are subject to risk, but also the estimate of the riskiness is typically subject to significant uncertainty. To incorporate such additional source of randomness into an asset pricing model, one has to introduce a second risk factor. This also allows to fit higher moments of the asset return distribution. In the traditional pricing approach mentioned above, one of the most prominent works in this direction is the Heston (1993) model. Based on an exogenous asset price process with stochastic volatility, explicit option pricing formulas are derived. The Heston (1993) model can be used successfully and allows also to be fitted to market data. However, empirical tests of the model in Bakshi et al. (1997) have shown that the 
fitted parameters are not consistent with parameters from time series data. This is economically unsatisfactory, since the model parameters loose their economic interpretation. In particular, the model demands for unreasonable levels of correlation and volatility of volatility to match the skewness and kurtosis effects observed in market option prices. Generally, an exogenously given price process seems unpleasant from an economic point of view, since any price process which cannot be supported by an economic model, lacks an economic justification and is subject to the objection of being arbitrary.

The focus of this paper is to derive analytical asset pricing formulas which impose as little as possible restrictions on the shape of the ASPK and include a second, correlated risk factor in the modelling of the information process. To this end, we consider an information process that is driven by a two-dimensional diffusion. Our approach is based on a class of ASPKs with declining elasticity. In this class of ASPKs, we derive analytical asset and option pricing formulas and determine credit spreads using a structural model approach.

Our approach should not be confused with the (also called) two-dimensional riskneutral valuation relationships in Franke et al. (2007). In fact, their approach is quite different from ours. They use a (declining elasticity) pricing kernel with two parameters, whereas we employ the generalized pricing kernels originally proposed in Lüders and Franke (2004). Moreover, Franke et al. (2007) propose a generalized lognormal distribution with two parameters. We use an information process driven by a two-dimensional diffusion with correlation, i.e. we introduce stochastic volatility as an additional risk factor. Then we derive explicit formulas for the case of a non-central chi-squared distribution of the volatility. We do not restrict ourselves to risk-neutral valuation relationships, but are interested in deriving explicit formulas to obtain a better understanding of the impact of investor preferences on asset pricing.

The contribution of our pricing model to the existing asset and option pricing literature consists of several aspects: The enhanced flexibility of our two-dimensional model allows for more accurate analytical and tractable asset and option pricing formulas. For example, our model provides an explanation for crash-like phenomena in time series of asset prices based on a rational expectations approach. It also seems to be a promising approach for pricing options on a stock index as the S\&P 500, since it allows to fit reasonable levels of kurtosis and skewness while using model parameters from time-series data. Credit spreads computed from our model in a simple structural model framework show quantitatively and qualitatively nice features. Moreover, our approach provides a convenient and more precise way to analyze the quantitative implications of non-constant elasticity of the ASPK. Also, since only the expected terminal distribution of the underlying needs to be known it presents an interesting model to price options when the underlying is not traded as, for example, with real options.

The paper is organized as follows. Section 2 presents the market model and the class of generalized ASPKs. In Section 3 the general valuation approach using the generalized characterization of the ASPK is shown. Based on this class of ASPKs, we derive analytical pricing formulas for assets and European options, when the final distribution at time $T$ is characterized by an information process that is driven by a two-dimensional diffusion with stochastic volatility. Furthermore, we determine 
credit spreads in a simple structural model. In Section 4, we investigate the influence of non-constant elasticity of the ASPK and stochastic volatility of the information process on asset and option prices and credit spreads. The paper is completed by a short conclusion.

\section{The model}

Throughout this paper we consider a market with a given time horizon $T>0$. We assume that the asset does not pay any dividends until terminal date $T$. To simplify the presentation we consider forward asset prices in this paper. Alternatively, one can assume the riskless interest rate to be zero. The fundamental asset pricing equation states that in an arbitrage free market the price of an asset is given by the expected future value of the asset, where the expectation is taken under some equivalent martingale measure $Q$. The equivalent martingale measure $Q$ is defined by

$$
Q(A)=\int_{A} \phi_{t, T} d P, \quad \forall A \in \mathscr{F}_{T},
$$

with the physical measure $P$ and the asset specific pricing kernel $\phi_{t, T}$. Given the equivalent martingale measure is defined by the ASPK $\phi_{t, T}$, the forward asset price $F_{t}$ for $0 \leq t \leq T$ can be written as

$$
F_{t}=\mathrm{E}^{Q}\left[I_{T}\right]=\mathrm{E}\left[I_{T} \phi_{t, T} \mid \mathscr{F}_{t}\right],
$$

where $I_{T}$ is the value of an information process at the terminal date $T$ and the filtration $\mathscr{F}_{t}$ characterizes the information available at time $0 \leq t \leq T$. Here and in the following $\mathrm{E}[\cdot]$ denotes the expected value with respect to the subjective measure $P$. The information process is exogenously given and defined as the conditional expectation of the terminal value of the underlying asset, i.e. $I_{t}=\mathrm{E}\left[F_{T} \mid \mathscr{F}_{t}\right]$. Due to the definition of the information process $I_{t}$, the value $I_{T}$ is equal to the terminal time $T$ value of the underlying. This may be either some liquidation value at time $T$ or simply the asset price at time $T$. Since the information process characterizes conditional expectations, it is a martingale; its drift is zero. Otherwise, the investor could improve his forecasts by anticipating the expected change in his forecasts. Assuming such an exogenous information process to model the information in the economy is common. The main advantages of this approach are that it is a parsimonious and intuitive way to characterize the filtration and that it has an economic interpretation, see Franke et al. (1999).

It follows that the price of a European call option with strike price $K$ and expiration date $T$ is given by

$$
C_{t}=\mathrm{E}\left[\max \left(I_{T}-K, 0\right) \phi_{t, T} \mid \mathscr{F}_{t}\right], \quad 0 \leq t \leq T .
$$

Throughout this paper, we will assume that the distribution of $I_{T}$ and the filtration $\left(\mathscr{F}_{t}\right)_{t \in[0, T]}$ are exogenously given. Our emphasis is to analyze the impact of the ASPK on asset prices, European option prices and credit spreads. 
We do not restrict ourselves to a single investor. Given a heterogeneous group of investors, however, it is known that their aggregation to a representative investor is non-trivial. Therefore, we here do not derive an equilibrium, and will simply assume the existence of a pricing kernel. Concerning the viability dicussion in two factor models we refer to Pham and Touzi (1996). They derive viability conditions from the representative investor's optimality condition and show that viability in the twofactor model implies that the pricing kernel is a deterministic function of time, wealth and volatility. Since we are interested in deriving explicit formulas, we neglect an additional dependence of the ASPK on volatility in our analysis. In particular, we follow Lüders and Franke (2004) who suggest to characterize the ASPK by

$$
\phi_{t, T}=\frac{\sum_{i=1}^{N} \alpha_{i} I_{T}^{\delta_{i}}}{\mathrm{E}\left[\sum_{i=1}^{N} \alpha_{i} I_{T}^{\delta_{i}} \mid \mathscr{F}_{t}\right]}, \quad 0 \leq t \leq T
$$

with $\alpha_{i}, \delta_{i} \in \mathbb{R}, N \in \mathbb{N} \cup\{\infty\}$. To generate arbitrage-free asset prices the only restriction which has to be imposed on the parameters is that $0<\phi_{t, T}<\infty, P$-a.s. This specification is rather general so that many different characteristics of the ASPK can be matched. Obviously the power function is a special case with $N=1$ in equation (1). This shape of the ASPK can either be related to a representative investor with declining relative risk aversion or to the aggregation of $N$ investors with power utility (see Düring and Lüders, 2005).

The flexibility of the ASPK is of great importance for option pricing but also for empirical investigations of option markets since recent empirical literature points to very complicated functional forms of empirical ASPKs. The main advantage of this class of ASPKs, besides the fact that very flexible shapes of the asset specific pricing kernel can be well approximated, is that these ASPKs are characterized by a series of non-central moments of the random variable. Hence, for different, one-dimensional distributions of the underlying asset, the ASPK and asset prices have been computed (Lüders and Franke, 2004) and analytical option pricing formulas have been derived (Düring and Lüders, 2005). Let us mention that in the literature there are also other parametric approaches to study the form of the ASPK, among others the orthogonal polynomial approach of Rosenberg and Engle (2002). Düring and Lüders (2005) provides a discussion of the technical aspects of characterization (1) as well as a comparison with other approaches to model the ASPK. Note that characterization (1) can also be further generalized to allow for more flexibility in time by allowing the coefficients to be functions of time rather than being constant, without affecting the main results of this paper. We give an example in Section 4.1. In the following section we derive analytical formulas for asset prices and European option prices based on this class of ASPK when the information process is a two-dimensional diffusion with stochastic volatility. We also consider credit spreads in a simple structural model framework. 


\section{Asset prices, options and credit spreads}

We consider a two-dimensional standard Brownian motion $W_{t}=\left(W_{t}^{(1)}, W_{t}^{(2)}\right)$ with correlation $d W_{t}^{(1)} d W_{t}^{(2)}=\rho d t$ on a given filtered probability space $\left(\Omega, \mathscr{F}, \mathscr{F}_{t}, P\right)$ where $\left(\mathscr{F}_{t}\right)_{t \in[0, T]}$ is the filtration generated by $W$ augmented by all the $\mathscr{F}$-null sets, with $\mathscr{F}=\mathscr{F}_{T}$. We assume that the value of the underlying asset at expiration is given by $I_{T}$ which is characterized by

$$
\begin{aligned}
d I_{t} & =\sqrt{\sigma_{t}} I_{t} d W_{t}^{(1)}, \\
d \sigma_{t} & =a\left(\sigma_{t}\right) d t+b\left(\sigma_{t}\right) d W_{t}^{(2)},
\end{aligned}
$$

for $0 \leq t \leq T$ with $I_{0}, \sigma_{0}>0$. Intuitively, such an information structure models the uncertainty about the "true" riskiness of $I_{T}$. Or, in other words, it accounts for the fact that not only the terminal value itself but also the amount of risk associated with it is unknown. Different choices for the drift coefficient $a\left(\sigma_{t}\right)$ and diffusion coefficient $b\left(\sigma_{t}\right)$ will lead to different models. By introducing a second risk factor this characterization of the information process is more flexible and realistic than the ones used previously in the literature. Through appropriate choices for the drift function $a\left(\sigma_{t}\right)$ it allows to introduce mean-reverting patterns of volatility 'shocks' which model information events like profit warnings.

\subsection{The general pricing methodology}

Our pricing methodology works in general as follows. In an arbitrage-free market the value of a European call (with expiration date $T$ ) at time $t \leq T$ is given by

$$
C\left(I_{t}, \sigma_{t}, t\right)=\mathrm{E}\left[\max \left(I_{T}-K, 0\right) \phi_{t, T} \mid \mathscr{F}_{t}\right] .
$$

Assume that the asset specific pricing kernel is characterized by equation (1) and define $\mu\left(t, \delta_{i}\right)=\mathrm{E}\left[I_{T}^{\delta_{i}} \mid \mathscr{F}_{t}\right]$. This yields

$$
\begin{aligned}
& C\left(I_{t}, \sigma_{t}, t\right)=\mathrm{E}\left[\max \left(\frac{\sum_{i=1}^{N} \alpha_{i} I_{T}^{\delta_{i}+1}}{\sum_{i=1}^{N} \alpha_{i} \mu\left(t, \delta_{i}\right)}-K \frac{\sum_{i=1}^{N} \alpha_{i} I_{T}^{\delta_{i}}}{\sum_{i=1}^{N} \alpha_{i} \mu\left(t, \delta_{i}\right)}, 0\right) \mid \mathscr{F}_{t}\right] \\
& =\int_{K}^{\infty} \frac{\sum_{i=1}^{N} \alpha_{i} I_{T}^{\delta_{i}+1}}{\sum_{i=1}^{N} \alpha_{i} \mu\left(t, \delta_{i}\right)} f\left(I_{T}, t\right) d I_{T}-K \int_{K}^{\infty} \frac{\sum_{i=1}^{N} \alpha_{i} I_{T}^{\delta_{i}}}{\sum_{i=1}^{N} \alpha_{i} \mu\left(t, \delta_{i}\right)} f\left(I_{T}, t\right) d I_{T} \\
& =\frac{\sum_{i=1}^{N}\left(\alpha_{i} \int_{K}^{\infty} I_{T}^{\delta_{i}+1} f\left(I_{T}, t\right) d I_{T}\right)}{\sum_{i=1}^{N} \alpha_{i} \mu\left(t, \delta_{i}\right)}-K \frac{\sum_{i=1}^{N}\left(\alpha_{i} \int_{K}^{\infty} I_{T}^{\delta_{i}} f\left(I_{T}, t\right) d I_{T}\right)}{\sum_{i=1}^{N} \alpha_{i} \mu\left(t, \delta_{i}\right)}
\end{aligned}
$$

where $f\left(I_{T}, t\right)$ is the conditional density function of $I_{T}$ where $\sigma_{T}$ is arbitrary. The price of a European put can be determined similarly or from the put-call parity. For the underlying asset this equation further simplifies to

$$
F_{t}=\mathrm{E}\left[\frac{\sum_{i=1}^{N} \alpha_{i} I_{T}^{\delta_{i}+1}}{\sum_{i=1}^{N} \alpha_{i} \mu\left(t, \delta_{i}\right)} \mid \mathscr{F}_{t}\right]=\frac{\sum_{i=1}^{N} \alpha_{i} \mu\left(t, \delta_{i}+1\right)}{\sum_{i=1}^{N} \alpha_{i} \mu\left(t, \delta_{i}\right)} .
$$


In the next two subsections we choose a specific form of the drift coefficient $a\left(\sigma_{t}\right)$ and the diffusion coefficient $b\left(\sigma_{t}\right)$. This allows us to derive analytical formulas for asset prices and European call options. Moreover, we determine credit spreads of corporate bonds using a structural model approach.

\subsection{Analytical formulas for asset and option prices}

In the following we choose the drift and diffusion coefficient in (2) to be

$$
a\left(\sigma_{t}\right)=\kappa\left(\theta-\sigma_{t}\right) \quad \text { and } \quad b\left(\sigma_{t}\right)=v \sqrt{\sigma_{t}},
$$

respectively, where $\kappa$ is the constant mean reversion speed, $\theta$ is the long-run mean of $\sigma_{t}$, and $v$ is the constant volatility of volatility. Hence, the volatility is non-central chi-squared distributed.

First, consider a European call option with strike price $K$ that expires at time $T$. Then, from equation (3) and the fact that $I_{T}$ is distributed according to (2) it follows by rearranging the terms that option prices in this ASPK class are given by

$$
\begin{aligned}
C\left(I_{t}, \sigma_{t}, t\right) & =\mathrm{E}\left[\max \left(I_{T}-K, 0\right) \frac{\sum_{i=1}^{N} \alpha_{i} I_{T}^{\delta_{i}}}{\mathrm{E}\left[\sum_{i=1}^{N} \alpha_{i} I_{T}^{\delta_{i}} \mid \mathscr{F}_{t}\right]} \mid \mathscr{F}_{t}\right] \\
& =\mathrm{E}\left[\sum_{i=1}^{N} \frac{\max \left(I_{T}-K, 0\right) \alpha_{i} I_{T}^{\delta_{i}}}{\mathrm{E}\left[\sum_{i=1}^{N} \alpha_{i} I_{T}^{\delta_{i}} \mid \mathscr{F}_{t}\right]} \mid \mathscr{F}_{t}\right] \\
& =\sum_{i=1}^{N} \frac{\mathrm{E}\left[\alpha_{i} I_{T}^{\delta_{i}} \mid \mathscr{F}_{t}\right]}{\mathrm{E}\left[\sum_{i=1}^{N} \alpha_{i} I_{T}^{\delta_{i}} \mid \mathscr{F}_{t}\right]} \mathrm{E}\left[\frac{\max \left(I_{T}-K, 0\right) \alpha_{i} I_{T}^{\delta_{i}}}{\mathrm{E}\left[\alpha_{i} I_{T}^{\delta_{i}} \mid \mathscr{F}_{t}\right]} \mid \mathscr{F}_{t}\right] .
\end{aligned}
$$

We prove in the appendix, that this can be understood as a weighted sum of option prices,

$$
C\left(I_{t}, \sigma_{t}, t\right)=\sum_{i=1}^{N} \omega_{i} C_{i}\left(F_{t}^{(i)}, \sigma_{t}, t ; K\right),
$$

where $\omega_{i}$ are weights and the prices $C_{i}$ are given by

$$
\mathrm{C}_{i}\left(F_{t}^{(i)}, \sigma_{t}, t ; K\right)=F_{t}^{(i)} \mathscr{I}_{1}-K \mathscr{I}_{2},
$$

with $(k=1,2)$

$$
\begin{aligned}
\mathscr{I}_{k} & =\frac{1}{2}+\frac{1}{\pi} \int_{0}^{\infty} \operatorname{Re}\left[\frac{e^{-i \xi \ln (K)} f_{k}(\xi)}{i \xi}\right] d \xi \\
f_{k}(\xi) & =e^{A\left(T-t, \xi ; \delta_{k}\right)-A\left(T-t, 0 ; \delta_{k}\right)+\sigma_{t}\left[B\left(T-t, \xi ; \delta_{k}\right)-B\left(T-t, 0 ; \delta_{k}\right)\right]+i \xi \ln I_{t}}, \\
A(\tau, \xi) & =\frac{\kappa \theta}{v^{2}}\left[(b+d) \tau-2 \ln \left(\frac{1-g e^{d \tau}}{1-g}\right)\right], \quad B(\tau, \xi)=\frac{b+d}{v^{2}} \frac{1-e^{d \tau}}{1-g e^{d \tau}} \\
g & =\frac{b+d}{b-d}, \quad d=\sqrt{\left(\xi^{2}+i \xi\left(1-2 \delta_{k}\right)+\delta_{k}\left(1-\delta_{k}\right)\right) v^{2}+b^{2}} \\
b & =\kappa-\rho v\left(i \xi+\delta_{k}\right), \quad \delta_{1}=\delta_{i}+1, \quad \delta_{2}=\delta_{i}
\end{aligned}
$$


The 'virtual asset' price $F_{t}^{(i)}$ is given by

$$
\begin{aligned}
F_{t}^{(i)} & =\frac{\mathrm{E}\left[I_{T}^{\delta_{i}+1} \mid \mathscr{F}_{t}\right]}{\mathrm{E}\left[I_{T}^{\delta_{i}} \mid \mathscr{F}_{t}\right]} \\
& =I_{t} e^{A\left(T-t, 0 ; \delta_{i}+1\right)-A\left(T-t, 0 ; \delta_{i}\right)+\sigma_{t}\left[B\left(T-t, 0 ; \delta_{i}+1\right)-B\left(T-t, 0 ; \delta_{i}\right)\right]}, \quad 0 \leq t \leq T .
\end{aligned}
$$

We call this a virtual asset price since $F_{t}^{(i)}$ is the price that would hold if the elasticity of the ASPK were $\delta_{i}$. The derivation of (7) is also given in the appendix.

To get a better understanding of equation (5), note that if $N=1$ and $\delta=-1$ then the first term in equation (5) is one and the option price is given by a formula, which looks similar as the Heston (1993, p.331) option pricing formula. However, recall that in the present situation the ASPK is given by a power function, while in the Heston case it is not. So the formulas do not coincide. If $N>1$ then the option price is a weighted sum of option prices, where every price $\mathrm{C}_{i}\left(F_{t}^{(i)}, \sigma_{t}, t ; K\right)$ corresponds to an economy with constant elasticity $\delta_{i}$. For very small levels of volatility of volatility, i.e. as $v \rightarrow 0$, the evolution of the volatility becomes deterministic. Hence, formula (5) approaches the values of the generalized Black-Scholes pricing formula derived in Düring and Lüders (2005) as $v \rightarrow 0$, with time-averaged volatility $\bar{\sigma}=\frac{1}{T-t} \int_{t}^{T} \sigma_{\tau} d \tau$. If, additionally, $N=1$ and $\delta=-1$, we recover the Black-Scholes formula.

The price of the underlying asset under the generalized ASPK is given by the weighted sum

$$
F_{t}=\sum_{i=1}^{N} \frac{\mathrm{E}\left[\alpha_{i} I_{T}^{\delta_{i}} \mid \mathscr{F}_{t}\right]}{\mathrm{E}\left[\sum_{i=1}^{N} \alpha_{i} I_{T}^{\delta_{i}} \mid \mathscr{F}_{t}\right]} F_{t}^{(i)}=\sum_{i=1}^{N} \omega_{i} F_{t}^{(i)}, \quad 0 \leq t \leq T .
$$

The proposed class of ASPKs therefore yields an analytical formulas for asset prices and for European options which are given by a weighted sum of generalized prices with stochastic volatility. Note that these formulas are written in terms of the expected terminal value $I_{t}$ of the underlying. This can be sometimes more convenient, when no market price for the underlying is available but the investors have some knowledge about the expected future payoff of the underlying. Moreover, pricing formulas depending explicitly on investors' expectations and on the parameters of the ASPK can be applied as a tool to study the impact of expectations and preferences on asset and option prices. In the case that asset prices are available, the observable price of the underlying is given by $(8)$.

\subsection{Credit spreads in a simple structural model}

The yield difference between corporate bonds and treasury bonds due to credit risk is called the credit spread. In this section we investigate credit spreads under the generalized ASPK and the dynamics of $I_{t}$ as given above. Here, we will only be concerned with the fraction of the credit spread that is inflicted by default risk. Other important factors like illiquidity and asymmetric taxation are not considered here. We develop a simple structural model. We assume that a firm issues a risky zero 
coupon bond $Z$ that can only default at maturity $T$. The bond defaults if the firm value at maturity is lower than a given default boundary $D$. We consider two different scenarios of investor compensation in case of default:

1. In case of default, bond holders receive a constant recovery rate $R \in(0,1)$. Under these assumptions we show in the appendix that the credit spread is given by

$$
y=-\frac{1}{T} \ln Z\left(I_{t}, \sigma_{t}, t\right)=-\frac{1}{T} \ln \left[1-(1-R) \sum_{i=1}^{N} \omega_{i} \mathrm{Z}_{i}\left(I_{t}, \sigma_{t}, t ; D\right)\right] .
$$

where each $\mathrm{Z}_{i}\left(I_{t}, \sigma_{t}, t ; D\right)$ is corresponding to an economy with constant elasticity $\delta_{i}$ and it holds

$$
\begin{aligned}
\mathrm{Z}_{i}\left(I_{t}, \sigma_{t}, t ; D\right) & =\frac{1}{2}+\frac{1}{\pi} \int_{0}^{\infty} \operatorname{Re}\left[\frac{e^{-i \xi \ln (D)} f(\xi)}{i \xi}\right] d \xi \\
f(\xi) & =e^{A\left(T-t, \xi ; \delta_{i}\right)-A\left(T-t, 0 ; \delta_{i}\right)+\sigma_{t}\left[B\left(T-t, \xi ; \delta_{i}\right)-B\left(T-t, 0 ; \delta_{i}\right)\right]+i \xi \ln I}, \\
A(\tau, \xi) & =\frac{\kappa \theta}{v^{2}}\left[(b+d) \tau-2 \ln \left(\frac{1-g e^{d \tau}}{1-g}\right)\right], \quad B(\tau, \xi)=\frac{b+d}{v^{2}} \frac{1-e^{d \tau}}{1-g e^{d \tau}} \\
g & =\frac{b+d}{b-d}, \quad d=\sqrt{\left(\xi^{2}+i \xi\left(1-2 \delta_{i}\right)+\delta_{i}\left(1-\delta_{i}\right)\right) v^{2}+b^{2}} \\
b & =\kappa-\rho v\left(i \xi+\delta_{i}\right)
\end{aligned}
$$

2. In case of default, bond holders receive a constant fraction $R \in(0,1)$ of the terminal firm value $F_{T}$. Under these assumptions it can be seen that the credit spread is given by

$$
y=-\frac{1}{T} \ln Z\left(I_{t}, \sigma_{t}, t\right)=-\frac{1}{T} \ln \left[1-(1-R) \sum_{i=1}^{N} \omega_{i} \mathrm{P}_{i}\left(I_{t}, \sigma_{t}, t ; D\right)\right],
$$

where $\mathrm{P}_{i}\left(I_{t}, \sigma_{t}, t ; D\right)$ are put option prices corresponding to an economy with constant elasticity $\delta_{i}$. For very small levels of volatility of volatility, i.e. as $v \rightarrow 0$, the evolution of the volatility becomes deterministic. Then, if $N=1$ and $\delta=-1$, the credit spreads of formula (10) approach the spreads of Merton (1974) with averaged volatility $\bar{\sigma}=\frac{1}{T-t} \int_{t}^{T} \sigma_{\tau} d \tau$.

We close this section with a note on the implementation of the above formulas. All formulas can easily be implemented and efficiently computed in real-time. However, there are two subtle points to note. First, the integrands appearing in the numerical integration which has to be performed can by highly oscillating. Therefore, the use of an adaptive integration method is recommended. We use an adaptive Gauß-Lobatto quadrature formula in our numerical simulations. The second issue is related to the complex logarithm appearing in the formulas. Standard algebraic software will typically return the principal value of the complex logarithm which has a branch cut along the negative real axis. If during numerical integration the argument in the complex logarithm crosses this axis, numerical problems can occur. Similar problems appear in the numerical implementation of the Heston (1993) option pricing formula. Different solutions to it have been suggested in the literature, cf. Kahl and Jäckel (2005) and Albrecher et al. (2007). 


\section{Effects of different pricing kernels}

\subsection{Asset prices with excess volatility}

To study the quantitative implications of non-constant elasticity of the ASPK and to get a better understanding of the influence of the model parameters, we first take a look at the price of the underlying, given by (8). Figure 1 shows plots of the underlying's price for different parameter sets for

- a two-term pricing kernel with declining elasticity with $N=2, \alpha_{1}=1, \alpha_{2}=5$, $\delta_{1}=-1, \delta_{2}=-10$, and for

- a standard pricing kernel with constant elasticity with $N=1, \alpha_{1}=1, \delta_{1}=-1$.

The left plot shows prices for different values of volatility $\sigma_{t}$ while the volatility of volatility $v=0.1$ is fixed. The right plot shows prices for different choices for the volatility of volatility $v$ while the actual volatility is kept constant at $\sigma_{t}=0.04$. In both plots, the time horizon is $T-t=3$ years, the long-run mean of volatility $\theta=0.04$ and other parameter values are as shown in Table 1 . Under the ASPK with constant elasticity the information and the asset price show a linear dependence. For the ASPK with declining elasticity the relationship becomes strongly nonlinear, with regions where an investor is only willing to pay a slightly higher price for the asset in spite of grown expectations on the asset's terminal value. On the other hand, there are regions, where the asset price that is rational under this investor preferences is rising fast although little additional information is provided. We observe that the price of the underlying is decreasing with increasing actual volatility $\sigma_{t}$. It also decreases when volatility of volatility $v$ is increased for both pricing kernels, although for the standard pricing kernel the differences are very small. Note that the plots display the asset price depending on the information level $I_{t}$ at the fixed time $T-t=3$. With time approaching maturity the 'hump-shaped' asset price will smoothly approach the identity, since at maturity asset price and information level coincide, i.e. $F_{T}=I_{T}$. To study the influence of the ASPK on the temporal evolution of asset prices we simulate (2) using Monte Carlo simulation. We compute times series on a daily basis using an Euler-Maruyama discretization. The time horizon is 3 years or 756 business days. For the current volatility and its long-run mean we use $\sigma_{t}=\theta=0.016641$. The other parameters are as shown in Table 1. Some sample time series are shown in Figure 2. Below each time series plot daily log returns of the information and the asset price process are shown.

Table 1: Default parameters for numerical simulations.

\begin{tabular}{lc}
\hline Parameter & Value \\
\hline correlation & $\rho=-0.28$ \\
volatility of volatility & $v=0.1$ \\
mean reversion speed & $\kappa=1.16$ \\
\hline
\end{tabular}



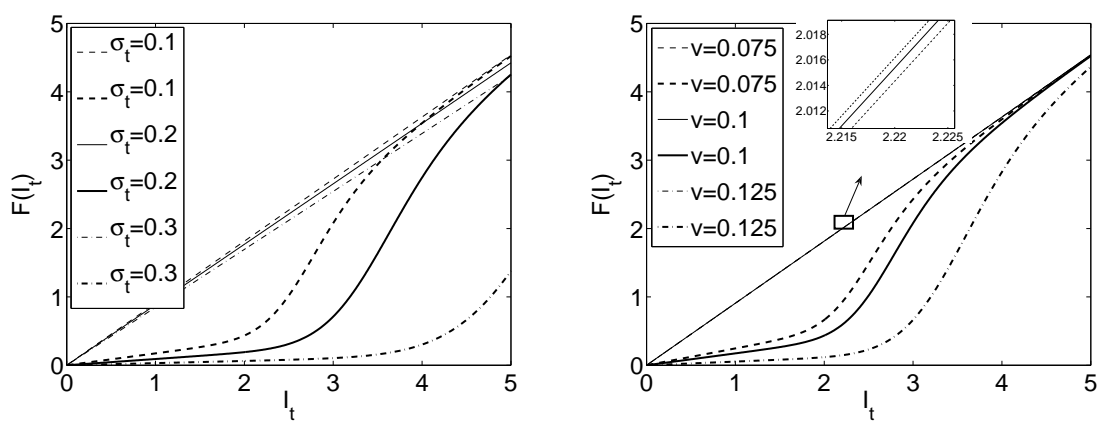

Fig. 1: Price $F_{t}$ of the underlying asset as a function of the information level $I_{t}$ for a two-term pricing kernel (thick lines, $N=2, \alpha_{1}=1, \alpha_{2}=5, \delta_{1}=-1, \delta_{2}=-10$ ) and for a standard pricing kernel with constant elasticity (thin lines, $N=1, \alpha_{1}=1$, $\delta_{1}=-1$.) The left figure shows prices for different values of volatility $\sigma_{t}$ and the right for different choices for the volatility of volatility $v$. The time to maturity is $T-t=3$ years and other parameter values are as shown in Table 1 .

The asset price $F_{t}$ is always smaller or equal than the value of the information process. Recall that $F_{t}$ is the forward asset price and we have chosen a riskless bond as numeraire. The gap between the forward asset price $F_{t}$ and the information process $I_{t}$, that quantifies the investors' current expectation of the terminal firm value, can be interpreted as a deduction for risk. It is larger in states with higher actual volatility and approaches zero as time approaches maturity $T$. When the information goes up, volatility is typically low, and the gap closes. The time series for the asset prices exhibit excess volatility and days with large negative returns although the information about the final firm value has only changed little. These negative shocks are a result of the stochastic volatility which is negatively correlated with the information process and the strongly nonlinear interplay between information and asset price process shown in Figure 1. Recall that our pricing model is based on rational expectations. The shock-like, large negative returns occur without exogenously added jumps in the underlying diffusion processes and are not result of learning or overconfidence effects. They are completely endogenously explained by rational behavior, more precisely by the investor's risk preference structure which is characterized by the shape of the ASPK. Our results are also in line with the theoretical findings of Franke et al. (1999), who show (for an information process following a geometric Brownian motion) that in the case of declining elasticity, the variance of the forward price increases relative to the constant elasticity case, and also that returns exhibit negative autocorrelation. 

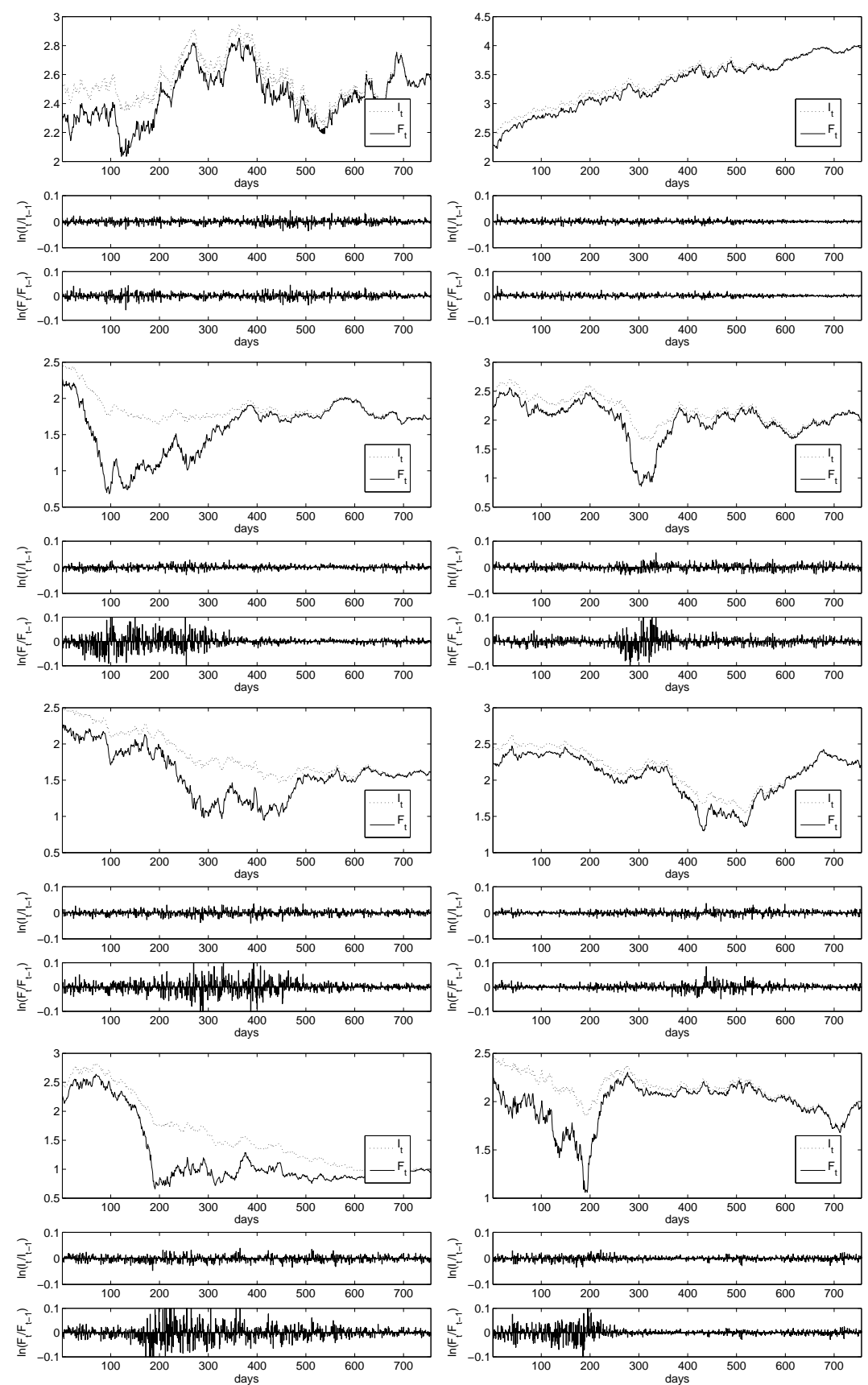

Fig. 2: Simulated time series for asset price $F_{t}$ and information $I_{t}$ for a two-term pricing kernel $\left(N=2, \alpha_{1}=1, \alpha_{2}=5, \delta_{1}=-1, \delta_{2}=-10\right)$. Logarithms of daily returns for $F_{t}$ and $I_{t}$ are shown below each graph. Time horizon is $T-t=3$ years (756 business days) and other parameter values are as shown in Table 1 except $\sigma_{0}=$ $\theta=0.016641$ 

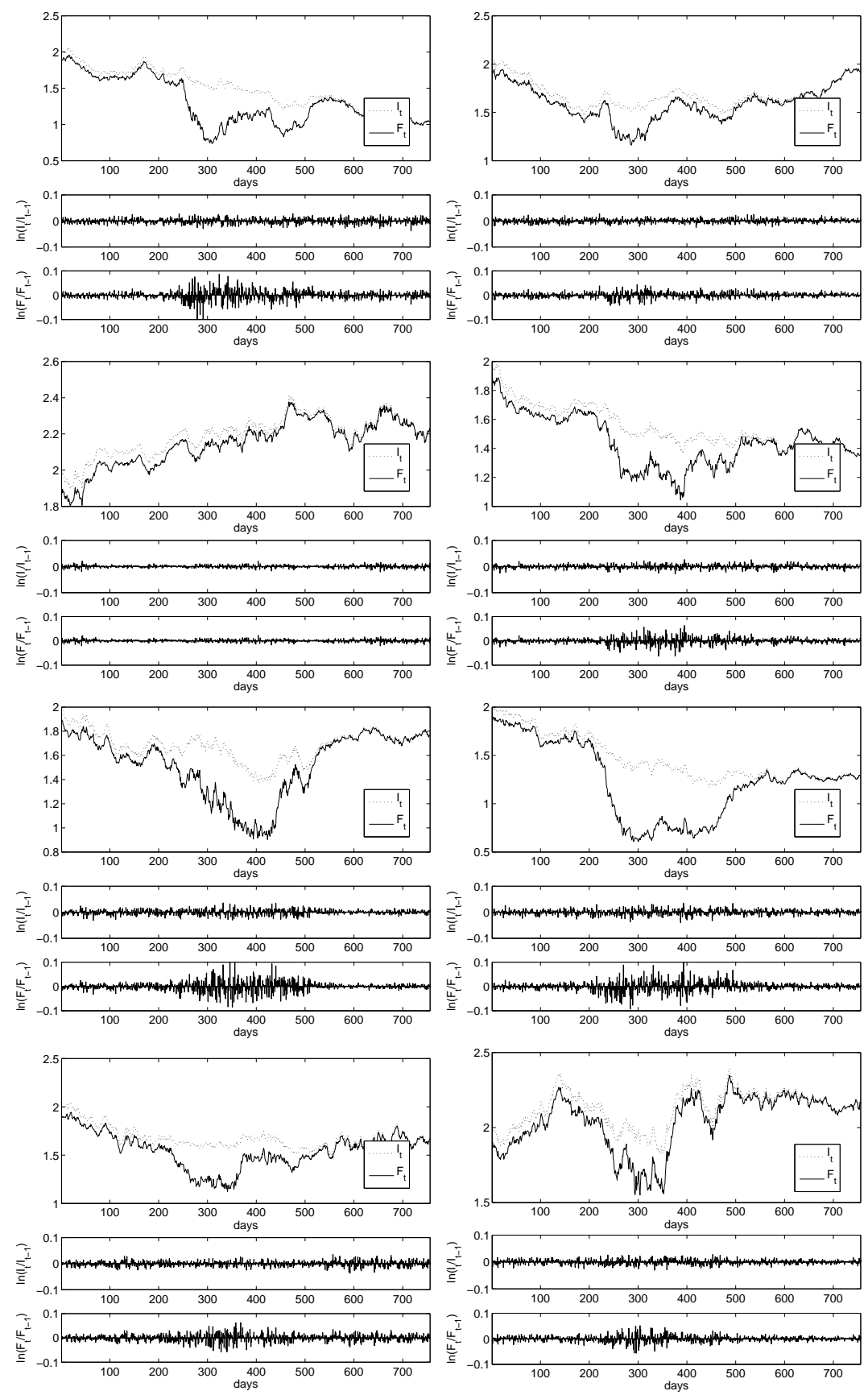

Fig. 3: Simulated time series for asset price $F_{t}$ and information $I_{t}$ for a time-dependent two-term pricing kernel $\left(N=2, \alpha_{1}=1, \alpha_{2}(t)=2.5[\tanh (10(t-1))+\tanh (-10(t-\right.$ $\left.2))], \delta_{1}=-1, \delta_{2}=-10\right)$. The ASPK switches smoothly from constant elasticity to declining elasticity and back. Logarithms of daily returns for $F_{t}$ and $I_{t}$ are shown below each graph. Time horizon is $T-t=3$ years (756 business days) and other parameter values are as shown in Table 1 except $\sigma_{0}=\theta=0.016641$. 
In reality, investor preferences will not be constant over time. They will change when certain exogenous information events, favorable or unfavorable, like profit warnings or forecasts of economic stagnation or recession occur. It is possible to incorporate such changes in investor preferences in our model by employing a timedependent ASPK. Since all integration in the pricing formulas are performed over the information variable, we can introduce time-dependent coefficients $\alpha_{i}(t), \delta_{i}(t)$ in the specification (1) of the ASPK.

To illustrate this, we repeat the simulation, but replace the time-constant ASPK by a time-dependent two-term ASPK with

$$
\alpha_{1}(t) \equiv 1, \alpha_{2}(t)=2.5[\tanh (10(t-1))+\tanh (-10(t-2))], \delta_{1}=-1, \delta_{2}=-10 .
$$

This ASPK changes smoothly from constant elasticity during the first year to an ASPK with declining elasticity during the second year and back. Some sample time series are shown in Figure 3. Below each time series plot daily log returns of the information and the asset price process are shown. We observe that during the first year and the third, where the ASPK has constant elasticity, the forward asset price $F_{t}$ is always close to the information level $I_{t}$. However, in the second year, when the investor changes his risk preference structure, decline in the information $I_{t}$ and accompanying high levels of volatility can lead to strong downward movements in the forward asset price.

\subsection{Option prices}

Next we turn to the effect of different ASPKs on the option price. We plot the price difference between the prices from formula (5) and Black-Scholes prices for different pricing kernels in Figure 4 using the parameter values from Table 1. These parameter choices are motivated by the time-series estimates that were obtained in Bakshi et al. (1997) from daily returns and volatility changes of the S\&P 500 index during a period from June 1988 to May 1991.

First, we use the standard pricing kernel with constant elasticity, i.e., we set $N=1$, $\alpha=1, \delta=-1$. The price differences compared to the Black-Scholes price displayed in Figure 4 show that call option prices are significantly higher in-the-money and lower out-of-the-money. For a two-term ASPK with declining elasticity $\left(N=2, \alpha_{1}=\right.$ $\left.1, \alpha_{2}=5, \delta_{1}=-1, \delta_{2}=-3,-5\right)$ we observe that the option prices are higher and the price difference is smaller out-of-the-money and more pronounced for in-the-money options. Such patterns are also found in empirical studies. The option prices increase for smaller values of $\delta$. This is consistent with Theorem 1 in Franke et al. (1999) who show that option prices are ceteris paribus higher under declining elasticity of the ASPK than under constant elasticity of the ASPK.

Stock return distributions observed in empirical studies show negative skewness and higher levels of kurtosis than explained by lognormal distribution used in the standard Black and Scholes (1973) option pricing formula. Alternative models have been proposed in the literature to address these issues. One of the most successful and widely accepted approaches is the stochastic volatility model of Heston (1993). However, empirical studies, e.g. Bakshi et al. (1997), show that the Heston (1993) 


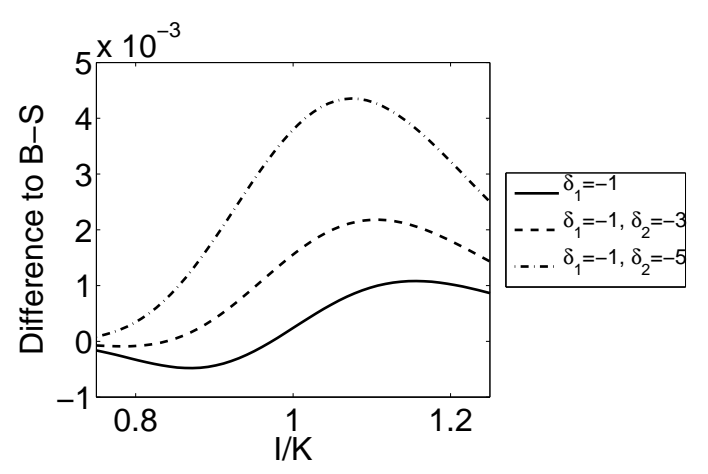

Fig. 4: Option price differences between prices from formula $(5)$ and the BlackScholes price for different pricing kernels: pricing kernel with constant elasticity $(N=1, \alpha=1, \delta=-1)$, and two term pricing kernel with declining elasticity $(N=2$, $\left.\alpha_{1}=1, \alpha_{2}=5, \delta_{1}=-1, \delta_{2}=-3,-5\right)$. Option prices are ceteris paribus higher under declining elasticity of the ASPK than under constant elasticity of the ASPK.

model demands for unreasonable levels of correlation $\rho$ and volatility of volatility $v$ to match the skewness and kurtosis effects observed in market option prices. The model seems to be misspecified, although this does not imply that the model cannot be applied successfully. In the following, we want to illustrate how the option pricing approach developed in the previous section can help to alleviate this shortcoming.

We compute the prices from the generalized prices from formula (5) and Heston (1993) prices. First, we use the standard pricing kernel with constant elasticity i.e. we set $N=1, \alpha=1, \delta=-1$. The price differences compared to the Black-Scholes price displayed in the left plot of Figure 5 show that call option prices are higher in-the-money and lower out-of-the-money. Prices are very similar as from the Heston (1993) formula. We used the same parameter values, current volatility $\sigma_{t}=0.04$, time to maturity $T-t=0.5$ and the other parameters as given in Table 1 for both models, although we increase the long run mean slightly from $\theta=0.04$ to $\theta=0.043$ for the Heston model so that both models match at the money.

Next, we would like to generate the strong negative skewness and high levels of kurtosis that are present in empirical data with both pricing formulas. Using the Heston model, we can achieve this by changing the parameters, for example, to

$$
\theta=0.07, \rho=-0.5, v=0.15,
$$

since these control the moments of the underlying distribution. Increasing these three values is what typically is necessary to match market option prices with the Heston (1993) formula. However, these parameter values are now no longer consistent with time-series estimates, e.g. such a strong negative correlation cannot be found in typical data. Interestingly, similar prices can be found using formula (5), if we use a twoterm ASPK with declining elasticity $\left(N=2, \alpha_{1}=1, \alpha_{2}=5, \delta_{1}=-1, \delta_{2}=-5.5\right)$ and still retain the more realistic parameter values used before. The price differences 

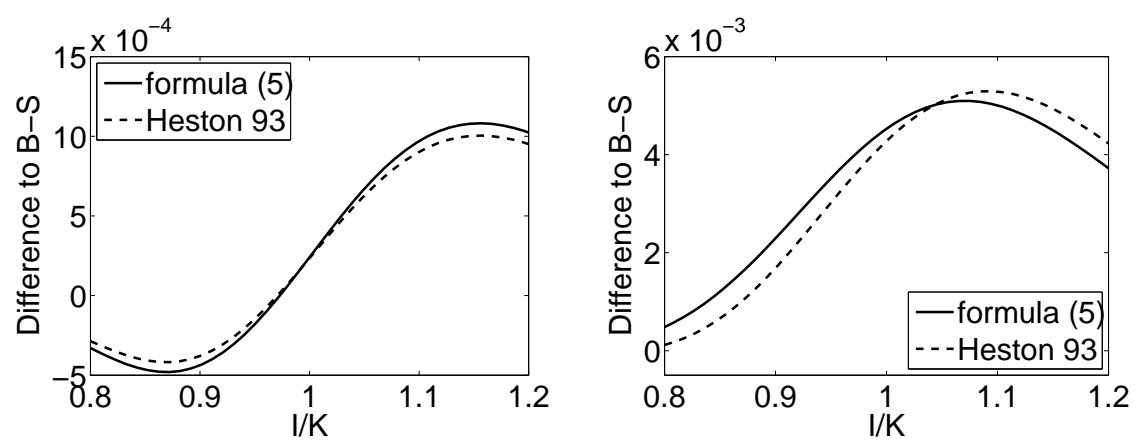

Fig. 5: Option price differences with respect to the Black-Scholes price of generalized prices from formula (5) and Heston (1993) prices for different pricing kernels: standard pricing kernel with constant elasticity $(N=1, \alpha=1, \delta=-1$, left), two term pricing kernel with declining elasticity $\left(N=2, \alpha_{1}=1, \alpha_{2}=5, \delta_{1}=-1\right.$, $\delta_{2}=-5.5$, right). To generate the negative skewness in the right plot, the parameters in the Heston (1993) model have to be set to unrealistic values that are no longer consistent with time-series data. For the generalized prices from formula (5) this is done by choosing an appropriate ASPK while retaining realistic model parameters.

compared to the Black-Scholes price are displayed in the right plot of Figure 5 for both approaches. The differences between the two approaches are small, and the qualitative behavior is very similar.

We also compute the implied volatilities of the option prices using the following iteration procedure. Let $\mathscr{C}$ be the option price computed by one of models and let $\sigma^{(0)}$ be a given starting value. Then,

- For a given volatility $\sigma^{(n)}$ compute the Black-Scholes option price $C\left(\sigma^{(n)}\right)$,

- Compute $\sigma^{(n+1)}=\sigma^{(n)}-\frac{C\left(\sigma^{(n)}\right)-\mathscr{C}}{C^{\prime}\left(\sigma^{(n)}\right)}$,

- Set $n:=n+1$, repeat cycle.

Let $\sigma_{i}^{(n)}$ denote the $n^{\text {th }}$ iterate of the implied volatility at grid point $I_{i}$. We stop the iteration procedure when the $l_{2}$ norm of the update defined by

$$
\varepsilon_{2}=\left(h \sum_{i=0}^{N}\left|\sigma_{i}^{(n+1)}-\sigma_{i}^{(n)}\right|^{2}\right)^{\frac{1}{2}}
$$

becomes less than $10^{-5}$.

Using the above setting we compute the implied volatilities for the two settings above: The Heston (1993) model with realistic and unrealistic parameters on one hand, and the pricing formula (5) with realistic parameters using the two different pricing kernels, one with constant, the other with declining elasticity. The results are shown in Figure 6. For the Heston model with realistic parameters from Table 1 and for the pricing formula (5) with the constant elasticity ASPK, the implied volatility is 
more or less flat (see left plot of Figure 6). Empirical data typically show a significant volatility skew, i.e. the implied volatility for in-the-money calls (i.e. out-of-the-money puts) is significantly higher than the implied volatility of at-the-money calls and outof-the-money calls. In the Heston model such a shape is obtained by increasing the parameters to the unrealistic values (11), see the right plot of Figure 6. For formula (5) using the two-term ASPK we find implied the volatility skew to be similar with higher volatilities for out-of-the-money calls.
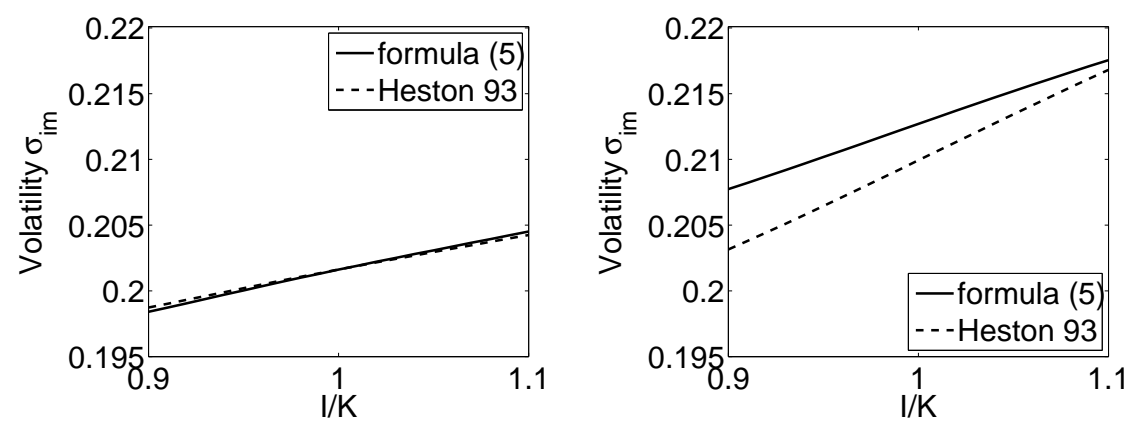

Fig. 6: The figure shows the implied volatilities for the two different settings: Heston model with realistic parameter values from Table 1 and the prices from formula (5) using a pricing kernel $(N=1, \alpha=1, \delta=-1)$ with constant elasticity (left plot), Heston (1993) model with unrealistic parameter values and the prices from formula (5) using a two term pricing kernel $\left(N=2, \alpha_{1}=1, \alpha_{2}=5, \delta_{1}=-1, \delta_{2}=-5.5\right.$ (right plot).

This illustrates, that using formula (5) with parameters from time-series data we can compute option prices that are consistent with empirical findings. Moreover, from a modeling point of view it has the advantage that the model parameters are clearly separated from the fitting parameters. The model parameters $\sigma_{0}, \theta, \rho, v, \kappa$ can be chosen from suitable estimates, e.g. of historic time-series data. To fit the model to the market, one can then choose suitable values of $\alpha_{i}, \delta_{i}$ in the specification (1) of the ASPK.

\subsection{Credit spreads}

Structural models for determining credit spreads (Merton, 1974; Longstaff and Schwartz, 1995) predict that the term structure of spreads is upward-sloping for high-grade bonds and downward-sloping for low-grade bonds. The intuition for a downwardslope is that low-grade bonds have a chance to upgrade. However, this is rejected by practitioners who claim that the term structure should always be upward sloping. The firm's leverage in the Merton (1974) model is implicitly falling since the firm value is drifting upwards at the riskless interest rate in the risk-neutral world. If 
one maintains a constant leverage ratio, this results in larger spreads and a upwardsloping term structure (Collin-Dufresne and Goldstein, 2001). This is e.g. justified by the assumption that firms issue additional debt or retire outstanding debt to maintain a target leverage ratio. In contrast, our model is based on the information process $I_{t}$ that reflects the investor's expectation of the terminal firm value. This information process has no drift, hence our model guarantees a constant leverage ratio implicitly. A second shortcoming of structural models is that the predicted spreads are too low. In our approach the size of the spreads is related to the investor preferences through the specific shape of the ASPK. To illustrate this, we report some results of numerical experiments we carried out.

Huang and Huang (2003) collected a number of historical data underlying their calibration study of different models. For B rated firms they report the average yield spread to be 470 basis points (bps) at 4 and 10 years. Based on their data we choose the leverage ratio to be 0.65 , i.e. we look at $\mathrm{B}$ rated bonds, and fix the recovery rate at $R=0.5$. We set $\sigma_{t}=\theta=0.016641$ and the other values as given in Table 1 . We use two ASPKs,

- a standard pricing kernel with constant elasticity with $N=1, \alpha_{1}=1, \delta_{1}=-1$, and $\delta_{2}=-10$, and

- a two-term pricing kernel declining elasticity with $N=2, \alpha_{1}=1, \alpha_{2}=5, \delta_{1}=$ $-1, \delta_{2}=-3.3$.

Then we compute the credit spreads using formula (9). The resulting credit spreads are shown in Figure 7.

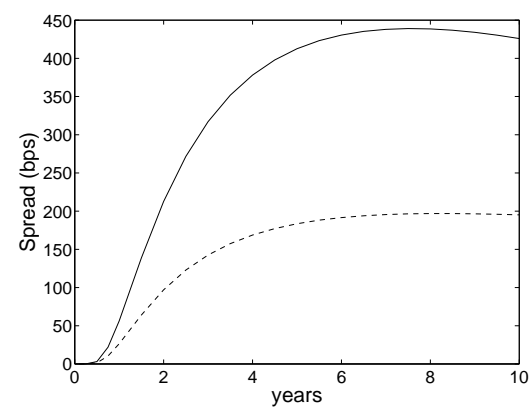

Fig. 7: The figure shows the credit spreads for B rated bonds computed from formula (9) using two different pricing kernels: one with constant elasticity (dashed line), the other with declining elasticity (solid line). The spreads are upward-sloping. Using the ASPK with declining elasticity they are of the order of historical estimates.

The spreads are upward sloping, unlike in the Merton (1974) model but in agreement with practitioner's claims. Under the ASPK with constant elasticity they are rather small and increase only slowly. Using the ASPK with declining elasticity however, they are show a steep ascent and then increase only moderately. The order of the spreads in this case agrees quite well with the historical estimates. 
To understand better the possibilities and limitations of our credit spread model it would be interesting to perform an empirical analysis. However, empirical studies of credit spreads using structural models are rather rare and are complicated by additional issues like coupon payment. On the other hand, the focus of this paper is on modelling investor risk preferences and understanding the interplay of the ASPK, risk preferences and asset and option prices and credit spreads. Therefore, we are rather interested in pricing methodology and modeling investor expectations and refrain from an empirical study which would be beyond the scope of this paper.

\section{Conclusion}

In this paper we presented a pricing approach for assets, European options and credit spreads based on a generalized specification of the asset specific pricing kernel (ASPK). The underlying information process is modelled by a two-dimensional diffusion. This rather general information process with stochastic volatility allowed us to derive analytical asset and option pricing formulas as well as to determine credit spreads in a simple structural model. The main observations can be summarized as follows. First, the asset prices in this rational expectations model show excess volatility and exhibit crash-like patterns in the asset price. Second, the resulting option pricing formula is consistent with the strong negative skewness and high levels of kurtosis observed in empirical studies. Third, the credit spreads derived in a simple structural model yield qualitatively and quantitatively promising results. Our approach may also be useful to infer empirical ASPK from option prices and credit spreads. As in existing parametric approaches it would allow to fit the ASPK to market data and could therefore be used for an empirical analysis of ASPKs.

\section{A Mathematical proofs and formulas}

\section{A.1 Asset price formula (7)}

First, we address the formula (7). The virtual asset price $F_{t}^{(i)}$ is given by

$$
F_{t}^{(i)}=\frac{\mathrm{E}\left[I_{T}^{\delta_{i}+1} \mid \mathscr{F}_{t}\right]}{\mathrm{E}\left[I_{T}^{\delta_{i}} \mid \mathscr{F}_{t}\right]} .
$$

Hence, we need to compute $V=\mathrm{E}\left[I_{T}^{\delta} \mid \mathscr{F}_{t}\right]$ for arbitrary $\delta$. Assuming that $V=V(I, \sigma, t)$ and sufficiently smooth, so that by Itô's lemma we obtain (note that in the following and in the rest of this section subscripts denote partial derivatives)

$$
d V=\left(V_{t}+\frac{1}{2} I^{2} \sigma V_{I I}+\rho b(\sigma) \sqrt{\sigma} I V_{I \sigma}+\frac{1}{2} b^{2}(\sigma) V_{\sigma \sigma}+a(\sigma) V_{\sigma}\right) d t+\sqrt{\sigma} I V_{I} d W^{(1)}+b(\sigma) V_{\sigma} d W^{(2)}
$$

Taking expectations on both sides we get the following partial differential equation

$$
V_{t}+\frac{1}{2} I^{2} \sigma V_{I I}+\rho b(\sigma) \sqrt{\sigma} I V_{I \sigma}+\frac{1}{2} b^{2}(\sigma) V_{\sigma \sigma}+a(\sigma) V_{\sigma}=0,
$$

which has to be solved for $I, \sigma>0,0<t<T$ with final condition $V(I, \sigma, T)=I^{\delta}$. Similar as in Heston (1993) we guess that the solution is of the form $V=I^{\delta} P$. Performing the transformation of variables $x=\ln I$ 
and $\tilde{t}=T-t$ (we immediately drop the tilde in the following) and substituting this functional form into (12), we arrive at

$$
\begin{aligned}
P_{t}-\frac{1}{2} \sigma\left[P_{x x}-P_{x}\right]-\delta \sigma P_{x}-\rho \sqrt{\sigma} b(\sigma) P_{x \sigma}- & \frac{1}{2} b^{2}(\sigma) P_{\sigma \sigma} \\
& -[a(\sigma)+\rho \sqrt{\sigma} b(\sigma) \delta] P_{\sigma}-\frac{1}{2} \sigma \delta(\delta-1) P=0,
\end{aligned}
$$

Introducing the Fourier transform of $P$,

$$
\hat{P}(\xi, \sigma, t)=\int_{\mathbb{R}} e^{i \xi x} P(x, \sigma, t) d x
$$

under which differentiation with respect to $x$ turns into multiplication with $-i \xi$, we obtain the following equation

$$
\begin{aligned}
\hat{P}_{t}=\frac{1}{2} b^{2}(\sigma) \hat{P}_{\sigma \sigma}+[a(\sigma)+\rho \sqrt{\sigma} b(\sigma) \delta-i \xi \rho \sqrt{\sigma} & b(\sigma)] \hat{P}_{\sigma} \\
& -\left[\frac{1}{2} \sigma \xi^{2}+i \xi\left(\delta-\frac{1}{2}\right) \sigma+\frac{1}{2} \delta(\delta-1) \sigma\right] \hat{P} .
\end{aligned}
$$

In the following we assume that $a(\sigma)=\kappa(\theta-\sigma)$ and $b(\sigma)=v \sqrt{\sigma}$. This allows us to solve explicitly for the characteristic function of $P$. Otherwise, one could resort to solve (14) numerically. By inserting the ansatz $\hat{P}=e^{A(t, \xi ; \delta)+\sigma B(t, \xi ; \delta)+i \xi x}$ with $A(0, \xi ; \delta)=B(0, \xi ; \delta)=0$ into (14) we get the two ordinary differential equations $\left({ }^{\prime}=\partial / \partial t\right)$

$$
\begin{aligned}
& A^{\prime}=\kappa \theta D, \\
& B^{\prime}=\frac{1}{2} v^{2} B^{2}-[\kappa-\rho v \delta-i \xi \rho v] B-\frac{1}{2} \xi^{2}+i \xi\left(\delta-\frac{1}{2}\right)+\frac{1}{2} \delta(\delta-1),
\end{aligned}
$$

subject to $A(0)=B(0)=0$, which can be solved yielding

$$
A=\frac{\kappa \theta}{v^{2}}\left[(b+d) t-2 \ln \left(\frac{1-g e^{d t}}{1-g}\right)\right], \quad B=\frac{b+d}{v^{2}} \frac{1-e^{d t}}{1-g e^{d t}},
$$

where

$$
g=\frac{b+d}{b-d}, \quad d=\sqrt{\left[\xi^{2}+i \xi(1-2 \delta)+\delta(1-\delta)\right] v^{2}+b^{2}}, \quad b=\kappa-\rho v(i \xi+\delta) .
$$

Note that since $P(x, \sigma, 0)=1$ it holds

$$
\hat{P}(\xi, \sigma, 0)=\int_{\mathbb{R}} e^{i \xi x} P(x, \sigma, 0) d x=2 \pi \delta_{0}(\xi),
$$

where $\delta_{0}(\cdot)$ is the delta distribution with zero mean. This can easily be seen from

$$
P(x, \sigma, 0)=\frac{1}{2 \pi} \int_{\mathbb{R}} e^{-i \xi x} \hat{P}(\xi, \sigma, 0) d \xi=\int_{\mathbb{R}} e^{-i \xi x} \delta_{0}(\xi) d \xi=e^{0}=1 .
$$

Hence, we obtain

$$
P(x, \sigma, T-t)=\int_{\mathbb{R}} \delta_{0}(\xi) e^{A(t, \xi ; \delta)+\sigma B(t, \xi ; \delta)+i \xi x} d \xi=e^{A(t, 0 ; \delta)+\sigma B(t, 0 ; \delta)} .
$$

Finally, we are able to compute the virtual asset price

$$
F_{t}^{(i)}=\frac{\mathrm{E}\left[I_{T}^{\delta_{i}+1} \mid \mathscr{F}_{t}\right]}{\mathrm{E}\left[I_{T}^{\delta_{i}} \mid \mathscr{F}_{t}\right]}=I_{t} e^{A\left(T-t, 0 ; \delta_{i}+1\right)-A\left(T-t, 0 ; \delta_{i}\right)+\sigma_{t}\left[B\left(T-t, 0 ; \delta_{i}+1\right)-B\left(T-t, 0 ; \delta_{i}\right)\right]} .
$$


A.2 Option pricing formula (5)

Now, we turn to formula (5). The term which is left to be computed is

$$
W(I, \sigma, t)=\mathrm{E}\left[I_{T}^{\delta} \max \left(I_{T}-K, 0\right) \mid \mathscr{F}_{t}\right]
$$

Applying Itô's lemma, $W(I, \sigma, t)$ has to solve (12) with final condition

$$
W(I, \sigma, T)=I^{\delta} \max (I-K, 0) .
$$

We make use of the ansatz

$$
W=I^{\delta}\left(I P_{1}-K P_{2}\right)
$$

with $P_{1,2}(I, \sigma, T)=\mathbf{1}_{I \geq K}$. Thus, $P_{1,2}$ can be interpreted as the conditional probabilities that the option expires in the money. Equation (12) is linear, hence both terms of (17) have to satisfy (12) separately. Applying the transformations as above and inserting each part separately into (12), we obtain two equations like (13) for $P_{1,2}$, where $\delta$ has to be replaced by $\delta+1$ in case of $P_{1}$. Performing now a complex Fourier transform (also called extended transform), i.e. $\xi \in \mathbb{C}$, we find that $P_{1,2}$ have to solve (14).

To continue, we need the transformed payoff given by

$$
\hat{P}_{1,2}(\xi, \sigma, 0)=\int_{\mathbb{R}} e^{i \xi x} P_{1,2}(x, \sigma, 0) d x=\int_{\mathbb{R}} e^{i \xi x} \mathbf{1}_{x \geq \ln K} d x=\int_{\ln K}^{\infty} e^{i \xi x} d x=\frac{-K^{i \xi}}{i \xi},
$$

with $x=\ln (I)$ and subject to $\operatorname{Im}(\xi)>0$. Inverting the characteristic function we obtain the desired probabilities

$$
\begin{aligned}
P_{1,2}(x, \sigma, T-t) & =\frac{1}{2 \pi} \int_{-\infty}^{\infty} \hat{P}_{1,2}(\xi, \sigma, 0) e^{A(t, \xi ; \delta)+\sigma B(t, \xi ; \delta)+i \xi x} d \xi \\
& =\frac{1}{2 \pi} \int_{-\infty}^{\infty} \frac{-K^{i \xi}}{i \xi} e^{A(t, \xi ; \delta)+\sigma B(t, \xi ; \delta)+i \xi x} d \xi \\
& =\frac{1}{2 \pi} \int_{-\infty}^{\infty} \frac{e^{-i \xi \ln K}}{i \xi} e^{A(t, \xi ; \delta)+\sigma B(t, \xi ; \delta)+i \xi x} d \xi \\
& =\frac{1}{2}+\frac{1}{\pi} \int_{0}^{\infty} \operatorname{Re}\left[\frac{e^{-i \xi \ln K}}{i \xi} e^{A(t, \xi ; \delta)+\sigma B(t, \xi ; \delta)+i \xi x}\right] d \xi,
\end{aligned}
$$

where $\delta=\delta_{i}+1$ in case of $P_{1}$, and $\delta=\delta_{i}$ for $P_{2}$. From

$$
W(I, \sigma, t)=\mathrm{E}\left[I_{T}^{\delta} \max \left(I_{T}-K, 0\right) \mid \mathscr{F}_{t}\right]=I^{\delta}\left(I P_{1}(x, \sigma, t)-K P_{2}(x, \sigma, t)\right)
$$

and from (16), we conclude that

$$
\begin{aligned}
\mathrm{E}\left[\frac{\max \left(I_{T}-K, 0\right) \alpha_{i} I_{T}^{\delta_{i}}}{\mathrm{E}\left[\alpha_{i} I_{T}^{\delta_{i}} \mid \mathscr{F}_{t}\right]} \mid \mathscr{F}_{t}\right] & =\frac{\mathrm{E}\left[\max \left(I_{T}-K, 0\right) I_{T}^{\delta_{i}} \mid \mathscr{F}_{t}\right]}{\mathrm{E}\left[I_{T}^{\delta_{i}} \mid \mathscr{F}_{t}\right]} \\
& =\frac{I_{t} P_{1}\left(\ln I_{t}, \sigma, t\right)-K P_{2}\left(\ln I_{t}, \sigma, t\right)}{P\left(\ln I_{t}, \sigma, t\right)} \\
& =F_{t}^{(i)} \mathscr{I}_{1}-K \mathscr{I}_{2},
\end{aligned}
$$

where $\mathscr{I}_{1}$ and $\mathscr{I}_{2}$ are given by (6) and $F_{t}^{(i)}$ by (7). Thus, formula (5) is proved. 


\section{A.3 Credit spreads}

We assume that the risky zero coupon bond $Z$ can only default at maturity $T$. The bond defaults if the firm value at maturity is lower than a given default boundary $D$. In case of default, bond holders receive a constant recovery rate $R \in(0,1)$. Thus, the bond's cash flow at maturity is given by

$$
Z\left(I_{T}, \sigma_{T}, T\right)=1-(1-R) \mathbf{1}_{I_{T}<D}
$$

In the spirit of Merton (1974), the bond price under the generalized ASPK is then obtained by

$$
\begin{aligned}
Z\left(I_{t}, \sigma_{t}, t\right) & =\mathrm{E}\left[\left(1-(1-R) \mathbf{1}_{I_{T}<D}\right) \phi_{t, T} \mid \mathscr{F}_{t}\right] \\
& =1-(1-R) \mathrm{E}\left[\mathbf{1}_{I_{T}<D} \phi_{t, T} \mid \mathscr{F}_{t}\right] \\
& \left.=1-(1-R) \mathrm{E}\left[\mathbf{1}_{I_{T}<D} \frac{\sum_{i=1}^{N} \alpha_{i} I_{T}^{\delta_{i}}}{\mathrm{E}\left[\sum_{i=1}^{N} \alpha_{i} I_{T}^{\delta_{i}} \mid \mathscr{F}_{t}\right]}\right] \mathscr{F}_{t}\right] \\
& =1-(1-R) \mathrm{E}\left[\sum_{i=1}^{N} \frac{\mathbf{1}_{I_{T}<D} \alpha_{i} I_{T}^{\delta_{i}}}{\mathrm{E}\left[\sum_{i=1}^{N} \alpha_{i} I_{T}^{\delta_{i}} \mid \mathscr{F}_{t}\right]} \mid \mathscr{F}_{t}\right] \\
& =1-(1-R) \sum_{i=1}^{N} \frac{\mathrm{E}\left[\alpha_{i} I_{T}^{\delta_{i}} \mid \mathscr{F}_{t}\right]}{\mathrm{E}\left[\sum_{i=1}^{N} \alpha_{i} I_{T}^{\delta_{i}} \mid \mathscr{F}_{t}\right]} \mathrm{E}\left[\frac{\mathbf{1}_{I_{T}<D} \alpha_{i} I_{T}^{\delta_{i}}}{\mathrm{E}\left[\alpha_{i} I_{T}^{\delta_{i}} \mid \mathscr{F}_{t}\right]} \mid \mathscr{F}_{t}\right] \\
& =1-(1-R) \sum_{i=1}^{N} \omega_{i} \mathrm{Z}_{i}\left(I_{t}, \sigma_{t}, t ; D\right),
\end{aligned}
$$

where $\omega_{i}$ are the weights and $Z_{i}\left(I_{t}, \sigma_{t}, t ; D\right)$ are corresponding to an economy with constant elasticity $\delta_{i}$. The $\mathrm{Z}_{i}\left(I_{t}, \sigma_{t}, t ; D\right)$ still need to be specified. To this end we need to compute $\mathrm{E}\left[\mathbf{1}_{I_{T}<D} I_{T}^{\delta} \mid \mathscr{F}_{t}\right]$ for arbitrary $\delta$. From Itô's lemma follows, that $Z_{i}$ solves (12) with final condition

$$
Z_{i}(I, \sigma, T)=I^{\delta} \mathbf{1}_{I_{T}<D} .
$$

We make use of the ansatz

$$
Z_{i}=I^{\delta} P_{3}
$$

with $P_{3}(I, \sigma, T)=\mathbf{1}_{I<D}$. Thus, $P_{3}$ is the conditional probability that default occurs. Applying the transformations as above, we obtain an equation like (13) for $P_{3}$. We continue as above and find that $P_{3}$ has to solve (14). Here, the transformed initial condition is given by

$$
\hat{P}_{3}(\xi, \sigma, 0)=\int_{\mathbb{R}} e^{i \xi x} P_{3}(x, \sigma, 0) d x=\int_{\mathbb{R}} e^{i \xi x} \mathbf{1}_{x<\ln D} d x=\frac{D^{i \xi}}{i \xi},
$$

with $x=\ln (I)$ and subject to $\operatorname{Im}(\xi)<0$. Inverting the characteristic function we obtain the desired probabilities

$$
\begin{aligned}
P_{3}(x, \sigma, T-t) & =\frac{1}{2 \pi} \int_{-\infty}^{\infty} \hat{P}_{3}(\xi, \sigma, 0) e^{A(t, \xi ; \delta)+\sigma B(t, \xi ; \delta)+i \xi x} d \xi \\
& =\frac{1}{2 \pi} \int_{-\infty}^{\infty} \frac{e^{i \xi \ln D}}{i \xi} e^{A(t, \xi ; \delta)+\sigma B(t, \xi ; \delta)+i \xi x} d \xi \\
& =\frac{1}{2}+\frac{1}{\pi} \int_{0}^{\infty} \operatorname{Re}\left[\frac{e^{i \xi \ln D}}{i \xi} e^{A(t, \xi ; \delta)+\sigma B(t, \xi ; \delta)+i \xi x}\right] d \xi .
\end{aligned}
$$

From

$$
Z_{i}(I, \sigma, t)=\mathrm{E}\left[I_{T}^{\delta} \mathbf{1}_{I_{T}<D} \mid \mathscr{F}_{t}\right]=I^{\delta} P_{3}(x, \sigma, t)
$$


and from (16), we conclude that

$$
\mathrm{Z}_{i}\left(I_{t}, \sigma_{t}, t ; D\right)=\mathrm{E}\left[\frac{\mathbf{1}_{I_{T}<D} \alpha_{i} I_{T}^{\delta_{i}}}{\mathrm{E}\left[\alpha_{i} I_{T}^{\delta_{i}} \mid \mathscr{F}_{t}\right]} \mid \mathscr{F}_{t}\right]=\frac{\mathrm{E}\left[\mathbf{1}_{I_{T}<D} I_{T}^{\delta_{i}} \mid \mathscr{F}_{t}\right]}{\mathrm{E}\left[I_{T}^{\delta_{i}} \mid \mathscr{F}_{t}\right]}=\frac{P_{3}\left(\ln I_{t}, \sigma, t\right)}{P\left(\ln I_{t}, \sigma, t\right)}
$$

Summing up, the $Z_{i}$ are given by

$$
\begin{aligned}
\mathrm{Z}_{i}\left(I_{t}, \sigma_{t}, t ; D\right) & =\frac{1}{2}+\frac{1}{\pi} \int_{0}^{\infty} \operatorname{Re}\left[\frac{e^{-i \xi \ln (D)} f(\xi)}{i \xi}\right] d \xi, \\
f(\xi) & =e^{A\left(T-t, \xi ; \delta_{i}\right)-A\left(T-t, 0 ; \delta_{i}\right)+\sigma_{t}\left[B\left(T-t, \xi ; \delta_{i}\right)-B\left(T-t, 0 ; \delta_{i}\right)\right]+i \xi \ln I}, \\
A(\tau, \xi) & =\frac{\kappa \theta}{v^{2}}\left[(b+d) \tau-2 \ln \left(\frac{1-g e^{d \tau}}{1-g}\right)\right], \quad B(\tau, \xi)=\frac{b+d}{v^{2}} \frac{1-e^{d \tau}}{1-g e^{d \tau}}, \\
g & =\frac{b+d}{b-d}, \quad d=\sqrt{\left(\xi^{2}+i \xi\left(1-2 \delta_{i}\right)+\delta_{i}\left(1-\delta_{i}\right)\right) v^{2}+b^{2}}, \\
b & =\kappa-\rho v\left(i \xi+\delta_{i}\right) .
\end{aligned}
$$

Thus, formula (18) is fully proved.

Writing the bond in terms of its yield to maturity, $Z=e^{-y T}$, the credit spread is given by

$$
y=-\frac{1}{T} \ln Z\left(I_{t}, \sigma_{t}, t\right)=-\frac{1}{T} \ln \left[1-(1-R) \sum_{i=1}^{N} \omega_{i} Z_{i}\left(I_{t}, \sigma_{t}, t ; D\right)\right] .
$$

Acknowledgements The author acknowledges support from the Deutsche Forschungsgemeinschaft, grant JU 359/6 (Forschergruppe 518). The author would like to thank Erik Lüders for fruitful discussions and Wolfgang Bühler and Günter Franke for helpful comments.

\section{References}

Ait-Sahalia, Y. and A.W. Lo. (2000). "Nonparametric Risk Management and Implied Risk Aversion," Journal of Econometrics 94, 9-51.

Albrecher, H., P. Mayer, W. Schoutens, and J. Tistaert (2007). “The little Heston trap," Wilmott, No.1, 83-92.

Bakshi, G., C. Cao, and Z. Chen (1997), "Empirical performance of alternative option pricing models," Journal of Finance 52(5), 2003-2049.

Benninga, S. and J. Mayshar. (2000). "Heterogeneity and Option Pricing," Review of Derivatives Research 4, 7-27.

Bick, A. (1987). "On the Consistency of the Black-Scholes Model with a General Equilibrium Framework," Journal of Financial and Quantitative Analysis 22, 259275.

Black, F. and M. Scholes (1973). "The Pricing of Options and Corporate Liabilities," Journal of Political Economy 81, 637-654.

Brennan, M.J. (1979). "The Pricing of Contingent Claims in Discrete Time Models," Journal of Finance 34, 53-68.

Câmara, A. (2003). "A Generalization of the Brennan-Rubinstein Approach for the Pricing of Derivatives," Journal of Finance 58, 805-819.

Câmara, A. (2005). "Option Prices Sustained by Risk-Preferences," Journal of Business 78, 1683-1708. 
Campbell, J.Y. and J.H. Cochrane (1999). "By Force of Habit: A Consumption-Based Explanation of Aggregate Stock Market Behavior," Journal of Political Economy 107, 205-251.

Cochrane, J.H. (2001). Asset Pricing, Princeton University Press.

Collin-Dufresne, P. and R. Goldstein (2001). "Do Credit Spreads reflect Stationary Leverage Ratios?,” Journal of Finance 56, 1928-12957.

Daniel, K., D. Hirshleifer, and A. Subrahmanyam (2001). "Overconfidence, Arbitrage, and Equilibrium Asset Pricing," Journal of Finance 56, 921-965.

Düring, B. and E. Lüders (2005). "Option prices under generalized pricing kernels," Review of Derivatives Research 8(2), 97-123.

Franke, G., R.C. Stapleton, and M.G. Subrahmanyam. (1999). "When are Options Overpriced? The Black-Scholes Model and Alternative Characterisations of the Pricing Kernel," European Finance Review 3, 79-102.

Franke, G., J. Huang, and R. Stapleton (2007). Two-dimensional risk-neutral valuation relationships for the pricing of options." Review of Derivatives Research 9(3), 213-237.

Heston, S.L. (1993). "A closed-form solution for options with stochastic volatility with applications to bond and currency options," Review of Financial Studies 6(2), 327-343.

Huang, J-Z. and M. Huang (2003). "How much of the Corporate-Treasury Yield Spread is Due to Credit Risk?," working paper.

Jackwerth, J.C. (2000). "Recovering Risk Aversion from Option Prices and Realized Returns," Review of Financial Studies 13, 433-451.

Kahl, C. and P. Jäckel (2005). "Not-so-complex logarithms in the Heston model", Wilmott, Sept. 2005, 94-103.

Karatzas, I. and S.E. Shreve (1991). Brownian Motion and Stochastic Calculus, New York, Springer.

Longstaff, F.A. and E.S. Schwartz (1995). "A Simple Approach to Valuing Risky Fixed and Floating Rate Debt," Journal of Finance 50, 789-819.

Lüders, E. and G. Franke (2004). "Predictability, Excess Volatility and Stock Market Crashes in Rational Expectations Models," working paper, CoFE discussion paper 04/05, University of Konstanz.

Merton, R.C. (1974). "On the pricing of corporate debt: The risk structure of interest rates." Journal of Finance 29, 449-470.

Pham, H. and N. Touzi (1996). "Equilibrium State Prices in a Stochastic Volatility Model”, Mathematical Finance 6, 215-236.

Rosenberg, J.V. and R.F. Engle (2002). "Empirical Pricing Kernels," Journal of Financial Economics 64, 341-732.

Rubinstein, M. (1976). The Valuation of Uncertain Income Streams and the Pricing of Options," Bell Journal of Economics and Management Science 7, 407-425.

Schroder, M. (2004). "Risk-Neutral Parameter Shifts and Derivatives Pricing in Discrete Time," Journal of Finance 59, 2375-2402.

Luiz Vitiello, L. and S. Poon (2006). "A General Equilibrium and Preference Free Model for Pricing Options Under Transformed Gamma Distribution,” working paper. 